\title{
Phosphorylation of Signal Transducer and Activator of Transcription 6 (STAT6) and STAT1, but Not STAT3, Induced by Antigen Inhalation in Bronchial Smooth Muscles of Sensitized Mice
}

\author{
Yoshihiko ChiBa, ${ }^{*}$ Michiko Todoroki, and Miwa Misawa \\ Department of Pharmacology, School of Pharmacy, Hoshi University; 2-4-41 Ebara, Shinagawa-ku, Tokyo 142-8501, \\ Japan. Received August 21, 2009; accepted October 13, 2009; published online October 15, 2009
}

The signal transducer and activator of transcription (STAT) family of molecules play a critical role in the signaling of many cytokines. In addition to STAT6, implication of STAT1 and STAT3 in the development of airway hyperresponsiveness (AHR) has also been suggested in allergic bronchial asthma. However, there is little information whether or not antigen challenge really causes the in vivo activation of these STAT molecules in bronchial smooth muscles (BSMs). In the present study, the activations of these STATs were examined in BSMs of mice with allergic bronchial asthma. Male BALB/c mice were sensitized and repeatedly challenged with ovalbumin (OA) antigen. Total protein samples of the left main bronchi were prepared at 3 after the last OA challenge, and Western blot analyses for total and tyrosine-phosphorylated STATs molecules were conducted. In addition to the phosphorylation of STAT6, a significant increase in the level of phosphorylated STAT1 was also observed after the antigen exposure. In contrast, no significant increase in the level of phosphorylated STAT3 was observed in this mouse model of allergic bronchial asthma. The antigen exposure did not change the protein expressions of these STATs themselves. These findings suggest that STAT6 and STAT1, but not STAT3, might be crucial signal transducers in the development of BSM hyperresponsiveness, one of the causes of AHR in asthmatics.

Key words allergic asthma; bronchial smooth muscle hyperresponsiveness; ovalbumin antigen; signal transducer and activator of transcription; mouse

The dramatic increase in the number of asthma cases over the last decades is of great concern for public health in the world. ${ }^{1)}$ The $\mathrm{CD}^{+} \mathrm{T}$ helper 2 lymphocytes (Th2 cells) are closely associated with disease severity, suggesting an integral role of Th2 cells in the pathophysiology of allergic bronchial asthma. ${ }^{2-5)}$ Th2 cells secrete various cytokines, termed Th2 cytokines, which cause several key features of allergic bronchial asthma, including airway hyperresponsiveness (AHR). ${ }^{2-5)}$ Increasing evidence indicates that interleukin-13 (IL-13), one of the members of Th2 cytokine family, is a crucial mediator in the development of AHR. ${ }^{6-8)}$ In addition, another Th2 cytokine IL-4 is also believed to play a role in asthma. ${ }^{9-11)}$ Interestingly, IL-4 shares many functional properties with IL-13, presumably because they share a common receptor composed of IL4Ra chain as one of the two hetero chains. ${ }^{12)}$

Most of the activities of IL-13 and IL-4 can be ascribed to the activation of signal transducer and activator of transcription 6 (STAT6). ${ }^{13-15)}$ Indeed, a critical role of the STAT6 signal transduction in the development of AHR has been suggested in STAT6-deficient mice. ${ }^{16,17)}$ Similarly, a cell-penetrating dominant-negative STAT6 peptide could inhibit AHR in a mouse model of allergic bronchial asthma. ${ }^{18)}$ On the other hand, recent studies also indicated an implication of the STAT family of molecules other than STAT6, such as STAT $1^{19)}$ and STAT3, ${ }^{20)}$ in the development of AHR. However, there is little information whether or not antigen challenge really causes the in vivo activation of these STAT molecules in bronchial smooth muscles (BSMs). In the present study, activations of these STAT molecules were examined in BSMs of a murine model of allergic asthma, which has marked BSM hyperresponsiveness. ${ }^{21,22)}$

\section{MATERIALS AND METHODS}

Animals Male BALB/c mice were purchased from the Charles River Japan, Inc. (Kanagawa, Japan) and housed in a pathogen-free facility. All animal experiments were approved by the Animal Care Committee of the Hoshi University (Tokyo, Japan).

Antigen Sensitization and Challenge Preparation of a murine model of allergic bronchial asthma was performed as described previously. ${ }^{21-25}$ ) In brief, BALB/c mice ( 8 weeks of age) were actively sensitized by intraperitoneal (i.p.) injections of $8 \mu \mathrm{g}$ ovalbumin (OA; Seikagaku Co., Tokyo, Japan) with $2 \mathrm{mg}$ Imject Alum (Pierce Biotechnology, Inc., Rockfold, IL, U.S.A.) on day 0 and day 5. The sensitized mice were challenged with aerosolized OA-saline solution $(5 \mathrm{mg} / \mathrm{ml})$ for $30 \mathrm{~min}$ on days 12,16 and 20 . A control group of mice received the same immunization procedure except for inhaling saline aerosol instead of $\mathrm{OA}$ challenge. The aerosol was generated with an ultrasonic nebulizer (Nihon Kohden, Tokyo, Japan) and introduced to a Plexiglas chamber box $(130 \times 200 \mathrm{~mm}, 100 \mathrm{~mm}$ height $)$ in which the mice were placed. Three hours after the last OA challenge, mice were sacrificed by exsanguination from the abdominal aorta under urethane $(1.6 \mathrm{~g} / \mathrm{kg}$, i.p.; Sigma-Aldrich, St. Louis, MO, U.S.A.) anesthesia.

Western Blot Analyses The immunoblot analyses were carried out as previously described. ${ }^{22,24)}$ In brief, protein samples of mouse left main bronchi were subjected to $7.5 \%$ sodium dodecyl sulfate-polyacrylamide gel electrophoresis (SDS-PAGE) and the proteins were then electrophoretically transferred to a polyvinylidene difluoride (PVDF) membrane. After blocking with $1 \%$ BlockAce $^{\mathrm{TM}}$ (Dainippon Sumitomo Pharma Co., Ltd., Osaka, Japan), the PVDF membrane was incubated with the primary antibody. The primary 
antibodies used in the present study were monoclonal mouse anti-STAT1 (1:1000 dilution; BD Biosciences, San Jose, CA, U.S.A.), anti-phospho-STAT1 (1:1000 dilution; BD Biosciences), anti-STAT3 (1:2500 dilution; BD Biosciences), anti-phospho-STAT3 (1:500 dilution; BD Biosciences), polyclonal rabbit anti-STAT6 (1:1000 dilution; Santa Cruz Biotechnology, Inc., Santa Cruz, CA, U.S.A.), and anti-phospho-STAT6 (1:1000 dilution; Santa Cruz Biotechnology, Inc.) antibodies. Then the membrane was incubated with horseradish peroxidase-conjugated donkey antirabbit immunoglobulin $\mathrm{G}$ (IgG) anti-mouse IgG (1 : 2500 dilution; Amersham Biosciences, Co., Piscataway, NJ, U.S.A.), detected by an enhanced chemiluminescent system (Amersham Biosciences, Co.) and analyzed by a densitometry system. Detection of house-keeping gene was also performed on the same membrane by using monoclonal mouse anti-glyceraldehyde-3-phosphate dehydrogenase (GAPDH) (1:10000 dilution; Chemicon International, Inc., Temecula, CA, U.S.A.) to confirm the same amount of proteins loaded.

Statistical Analyses All the data were expressed as the mean with S.E. Statistical significance of difference was determined by two-way analysis of variance (ANOVA) with post hoc Bonferroni/Dunn (StatView for Macintosh ver. 5.0, SAS Institute, Inc., NC, U.S.A.). A value of $p<0.05$ was considered significant.

\section{RESULTS}

Phosphorylation of STAT6 in BSMs of Mice Induced by Antigen Exposure In the present study, we used our well-established murine model of allergic bronchial asthma. $^{21-25)}$ In our previous study that used the whole lung homogenates as protein samples, the time-course examination revealed that a peak of the STAT6 phosphorylation was observed at $3 \mathrm{~h}$ after the ovalbumin (OA) inhalation (Chiba et al., 2009). So currently, the phosphorylated STATs in BSMs
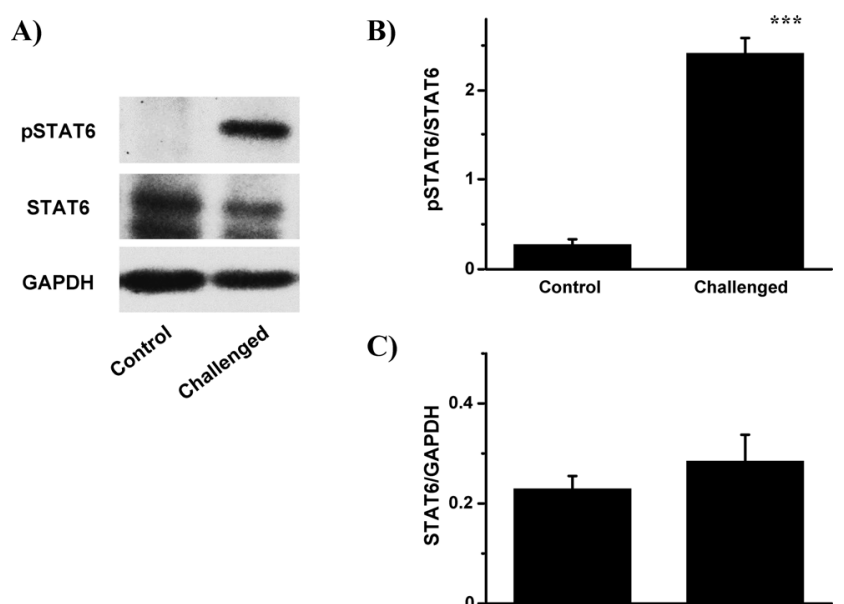

C)

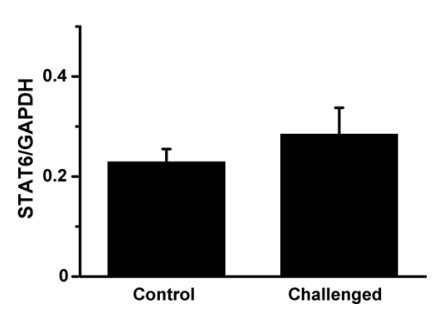

Fig. 1. Change in the Level of Phosphorylated Signal Transducer and Activator of Transcription 6 (STAT6) in Lungs after Challenge with Ovalbumin (OA) Antigen

Male BALB/c mice were actively sensitized and repeatedly challenged with OA as described in Materials and Methods. The protein samples of bronchial smooth muscles were subjected to immunoblot analyses. (A) Typical immunoblots of phosphorylated STAT6 (pSTAT6; upper), total STAT6 (middle) and GAPDH (lower). The levels of pSTAT6/STAT6 (B) and STAT6/GAPDH (C) were calculated. Each column represents the mean \pm S.E. from 6 different animals. $* * * p<0.001 v s$. control by Bonferroni/Dunn test. were analyzed at $3 \mathrm{~h}$ after the OA challenge. As shown in Fig. 1, the OA challenge caused a marked and significant increase in the level of phosphorylated STAT6 in the BSM tissues (Figs. 1A, B). On the other hand, the antigen exposure did not affect the expression of STAT6 protein itself (Fig. 1C).

Phosphorylation of STAT1, but Not STAT3, in BSMs of Mice Induced by Antigen Exposure Utilizing the identical protein samples used for the STAT6 phosphorylation analyses, the levels of phosphorylated STAT1 and STAT3 were also measured by immunoblottings. As well as the case of STAT6, a peak of the STAT1 phosphorylation in the whole
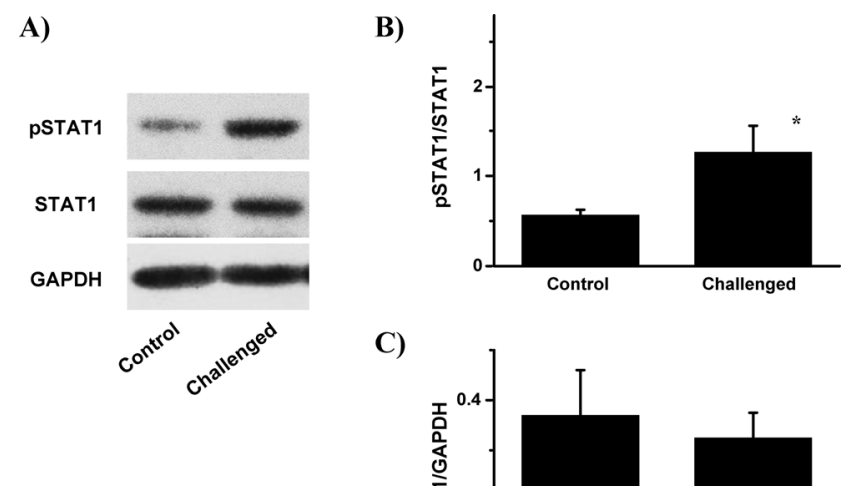

C)

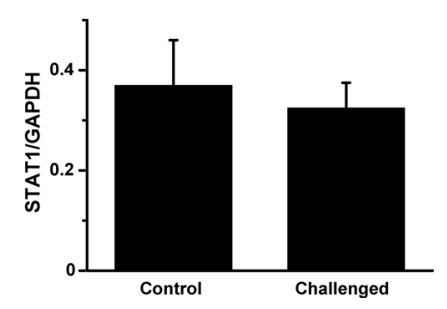

Fig. 2. Change in the Level of Phosphorylated Signal Transducer and Activator of Transcription 1 (STAT1) in Lungs after Challenge with Ovalbumin (OA) Antigen

Male BALB/c mice were actively sensitized and repeatedly challenged with OA as described in Materials and Methods. The protein samples of bronchial smooth muscles were subjected to immunoblot analyses. (A) Typical immunoblots of phosphorylated STAT1 (pSTAT1; upper), total STAT1 (middle) and GAPDH (lower). The levels of pSTAT1/STAT1 (B) and STAT1/GAPDH (C) were calculated. Each column represents the mean \pm S.E. from 6 different animals. $* p<0.05 v s$. control by Bonferroni/Dunn test.

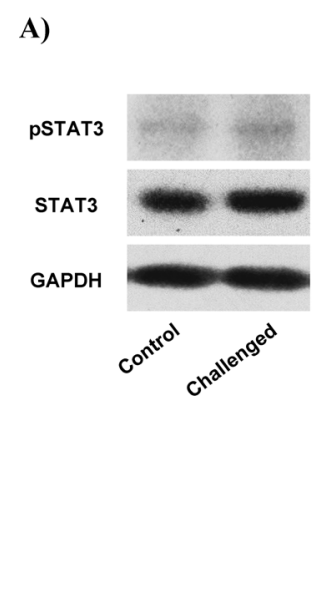

B)

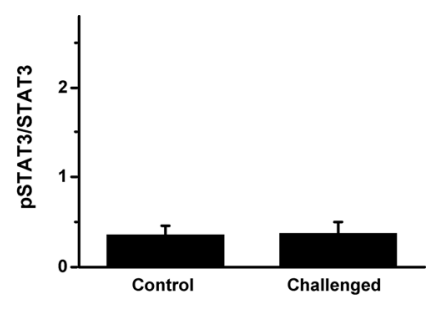

C)

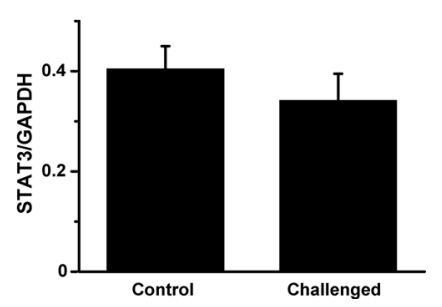

Fig. 3. Change in the Level of Phosphorylated Signal Transducer and Activator of Transcription 3 (STAT3) in Lungs after Challenge with Ovalbumin (OA) Antigen

Male BALB/c mice were actively sensitized and repeatedly challenged with $\mathrm{OA}$ as described in Materials and Methods. The protein samples of bronchial smooth muscles were subjected to immunoblot analyses. (A) Typical immunoblots of phosphorylated STAT3 (pSTAT3; upper), total STAT3 (middle) and GAPDH (lower). The levels of pSTAT3/STAT3 (B) and STAT3/GAPDH (C) were calculated. Each column represents the mean \pm S.E. from 6 different animals 
lung homogenates was observed at $3 \mathrm{~h}$ after the OA inhalation (unpublished observation). As shown in Fig. 2A, a distinct phosphorylation of STAT1 in lung homogenate was detected after the OA antigen challenge. The level of phosphorylated STAT1 (Fig. 2B), but not total STAT1 protein (Fig. 2C), was significantly increased by the antigen challenge. On the other hand, however, no significant increase in the level of pSTAT3 was observed by the antigen exposure, although STAT3 protein was distinctly expressed in the BSMs used (Fig. 3). Consistently, no significant increase in the level of pSTAT3 in the whole lung homogenates was observed at any time points measured (unpublished observation).

\section{DISCUSSION}

In the present study, the activations of STAT6, STAT1 and STAT3 in BSMs of mice with allergic bronchial asthma were examined by immunoblottings using antibodies against phosphorylated these molecules. In addition to the phosphorylation of STAT6 (Fig. 1), a major signal transducer activated by Th2 cytokines such as IL-13 and IL-4 in allergic bronchial asthma, ${ }^{13-15)}$ we show here for the first time that STAT1 is also phosphorylated in BSMs after the inhalation of OA antigen (Fig. 2). In contrast, no significant increase in the level of phosphorylated STAT3 was observed in this mouse model of the disease (Fig. 3). It is thus possible that activations of STAT6 and STAT1, but not STAT3, might be involved in the development of BSM hyperresponsiveness, one of the causes of the AHR in asthmatics.

Airway smooth muscle is an important effector tissue regulating bronchomotor tone. It has been suggested that one of the factors that contribute to the AHR is an abnormality of the properties of airway smooth muscle. ${ }^{26,27)}$ In agreement with our previous study, ${ }^{22}$ the level of phosphorylated STAT6 was significantly increased in BSMs after the antigen exposure (Fig. 1). There is increasing evidence that activation of STAT6 is a key in the development of AHR in allergic bronchial asthma. In allergic asthmatics, the level of phosphorylated STAT6 was increased in peripheral $\mathrm{CD}^{+}$ CD161 ${ }^{+}$T cells. $^{28)}$ An increase in phosphorylated STAT6 in the airways was also observed after allergen inhalation in atopic asthma. ${ }^{29)}$ In mouse models of allergic bronchial asthma, development of AHR was inhibited by STAT6 gene knockout ${ }^{16,17)}$ and by the treatment with a STAT6 inhibitory peptide $^{18)}$ or a short interfering RNA against STAT6. ${ }^{30)}$

In addition to the phosphorylation of STAT6, a significant increase in the level of phosphorylated STAT1 was also found in BSMs of the antigen-challenged mice (Fig. 2). The activation of STAT1 might also be implicated in the pathogenesis of allergic airway diseases. Sampath and colleagues $^{31)}$ provided the first evidence for a possible role of STAT1 in allergic bronchial asthma. They found that phosphorylated STAT1 was increased in airway epithelial cells of asthmatic patients. ${ }^{31)}$ In a mouse model of allergic bronchial asthma, Quarcoo and colleagues ${ }^{19)}$ reported that local application of decoy oligonucleotide specific for STAT1 into airways could attenuate antigen-induced airway inflammation and hyperresponsiveness. More recently, Hattori and colleagues $^{32)}$ found that STAT1-deficient mice failed to develop inflammation and hyperresponsiveness of nasal airways induced by topical antigen challenge. In contrast, however, a recent study raised a possibility that the activated STAT1 may have an ability to inhibit the expression of RhoA, ${ }^{33)}$ an important protein responsible for the AHR. ${ }^{21,22,24,34-38)}$ Detailed studies are required to make clear the role of STAT1 in the development of AHR.

In conclusion, the current study clearly showed that STAT6 and STAT1, but not STAT3, were phosphorylated by antigen exposure in BSM tissues of sensitized mice. In addition to the importance of STAT6, the activation of STAT1 might also be involved in the development of BSM hyperresponsiveness, one of the causes of AHR in allergic bronchial asthma.

Acknowledgments This work was supported by the Ministry of Education, Culture, Sports, Science and Technology of Japan, and The Pharmacological Research Foundation, Tokyo, Japan. We thank Yuichi Nishida for his technical assistance.

\section{REFERENCES}

1) Eder W., Ege M. J., von Mutius E., N. Engl. J. Med., 355, 2226-2235 (2006).

2) Bochner B. S., Undem B. J., Lichtenstein L. M., Annu. Rev. Immunol., 12, 295-335 (1994).

3) Cohn L., Elias J. A., Chupp G. L., Annu. Rev. Immunol., 22, 789-815 (2004).

4) Robinson D. S., Hamid Q., Ying S., Tsicopoulos A., Barkans J., Bentley A. M., Corrigan C., Durham S. R., Kay A. B., N. Engl. J. Med., 326, 298-304 (1992).

5) Walker C., Kaegi M. K., Braun P., Blaser K., J. Allergy Clin. Immunol., 88, 935-942 (1991).

6) Grunig G., Warnock M., Wakil A. E., Venkayya R., Brombacher F., Rennick D. M., Sheppard D., Mohrs M., Donaldson D. D., Locksley R. M., Corry D. B., Science, 282, 2261-2263 (1998).

7) Wills-Karp M., Luyimbazi J., Xu X., Schofield B., Neben T. Y., Karp C. L., Donaldson D. D., Science, 282, 2258-2261 (1998).

8) Wills-Karp M., Immunol. Rev., 202, 175-190 (2004).

9) Bryborn M., Adner M., Cardell L. O., Clin. Exp. Allergy, 34, 12911298 (2004).

10) Dabbagh K., Takeyama K., Lee H. M., Ueki I. F., Lausier J. A., Nadel J. A., J. Immunol., 162, 6233-6237 (1999).

11) Steinke J. W., Borish L., Respir. Res., 2, 66-70 (2001).

12) Callard R. E., Matthews D. J., Hibbert L., Immunol. Today, 17, 108110 (1996).

13) Chatila T. A., Trends Mol. Med., 10, $493-499$ (2004).

14) Hebenstreit D., Wirnsberger G., Horejs-Hoeck J., Duschl A., Cytokine Growth Factor Rev., 17, 173-188 (2006).

15) Kuperman D. A., Schleimer R. P., Curr. Mol. Med., 8, 384-392 (2008).

16) Akimoto T., Numata F., Tamura M., Takata Y., Higashida N., Takashi T., Takeda K., Akira S., J. Exp. Med., 187, 1537-1542 (1998).

17) Kuperman D., Schofield B., Wills-Karp M., Grusby M. J., J. Exp. Med., 187, 939-948 (1998).

18) McCusker C. T., Wang Y., Shan J., Kinyanjui M. W., Villeneuve A., Michael H., Fixman E. D., J. Immunol., 179, 2556-2564 (2007).

19) Quarcoo D., Weixler S., Groneberg D., Joachim R., Ahrens B., Wagner A. H., Hecker M., Hamelmann E., J. Allergy Clin. Immunol., 114, 288-295 (2004).

20) Simeone-Penney M. C., Severgnini M., Tu P., Homer R. J., Mariani T. J., Cohn L., Simon A. R., J. Immunol., 178, 6191-6199 (2007).

21) Chiba Y., Ueno A., Shinozaki K., Takeyama H., Nakazawa S., Sakai H., Misawa M., Respir. Res., 6, Art. No. 4 (2005).

22) Chiba Y., Todoroki M., Nishida Y., Tanabe M., Misawa M., Am. J. Respir. Cell Mol. Biol., 41, 516-524 (2009).

23) Chiba Y., Kurotani R., Kusakabe T., Miura T., Link B. W., Misawa M., Kimura S., Am. J. Respir. Crit. Care Med., 173, 958-964 (2006).

24) Chiba Y., Nakazawa S., Todoroki M., Shinozaki K., Sakai H., Misawa M., Am. J. Respir. Cell Mol. Biol., 40, 159-167 (2009).

25) Chiba Y., Onoda S., Hattori Y., Maitani Y., Sakai H., Misawa M., 
Lung, 187, 179-185 (2009).

26) Martin J. G., Duguet A., Eidelman D. H., Eur. Respir. J., 16, 349-354 (2000).

27) Seow C. Y., Schellenberg R. R., Pare P. D., Am. J. Respir. Crit. Care Med., 158, S179-S186 (1998).

28) Gernez Y., Tirouvanziam R., Nguyen K. D., Herzenberg L. A., Krensky A. M., Nadeau K. C., J. Allergy Clin. Immunol., 120, 1441-1448 (2007).

29) Phipps S., Benyahia F., Ou T. T., Barkans J., Robinson D. S., Kay A. B., Am. J. Respir. Cell Mol. Biol., 31, 626-632 (2004).

30) Darcan-Nicolaisen Y., Meinicke H., Fels G., Hegend O., Haberland A., Kühl A., Loddenkemper C., Witzenrath M., Kube S., Henke W., Hamelmann E., J. Immunol., 182, 7501-7508 (2009).

31) Sampath D., Castro M., Look D. C., Holtzman M. J., J. Clin. Invest.,
103, 1353-1361 (1999).

32) Hattori H., Rosas L. E., Okano M., Durbin J. E., Nishizaki K., Satoskar A. R., Am. J. Rhinol., 21, 241-247 (2007).

33) Wang S., Koromilas A. E., Cell Cycle, 8, 2070-2079 (2009).

34) Chiba Y., Takada Y., Miyamoto S., Mitsui-Saito M., Karaki H., Misawa M., Br. J. Pharmacol., 127, 597-600 (1999).

35) Chiba Y., Goto K., Hirahara M., Sakai H., Misawa M., J. Pharmacol. Sci., 106, 615-625 (2008).

36) Gosens R., Schaafsma D., Nelemans S. A., Halayko A. J., Mini Rev. Med. Chem., 6, 339-348 (2006).

37) Kume H., Curr. Med. Chem., 15, 2876-2885 (2008).

38) Schaafsma D., Roscioni S. S., Meurs H., Schmidt M., Cell. Signal., 20, 1705-1714 (2008). 